\title{
Thin classes of separating sets
}

\author{
Reed Solomon
}

\begin{abstract}
There are various definitions for a Martin-Pour-El theory in the literature. We isolate two such definitions: weak Martin-Pour-El theories (which correspond to perfect thin $\Pi_{1}^{0}$ classes) and strong Martin-Pour-El theories (which correspond to thin classes of separating sets). By concentrating on constructions of appropriate $\Pi_{1}^{0}$ classes, rather than on direct constructions of the theories, we show how the extend versions of well known results about weak Martin-Pour-El theories to strong Martin-Pour-El theories. In addition, we consider how the degrees of complete consistent extensions of a strong Martin-Pour-El theory relate to the degree of the theory. Finally, we give a new restriction on the degrees of sets occurring in a thin $\Pi_{1}^{0}$ class and prove that this restriction is strictly stronger than previously known results.
\end{abstract}

\section{Introduction}

$\Pi_{1}^{0}$ classes can be used to represent many different collections of objects in mathematics, including the set of complete consistent extensions of a computably enumerable (c.e.) theory. We begin with a brief discussion of this connection. The reader who is not familiar with $\Pi_{1}^{0}$ classes and their connections to c.e. theories is referred to Cenzer $[\mathbf{C}]$ and Cenzer and Remmel $[\mathbf{C R}]$ for a more detailed introduction.

A tree is a set $T \subseteq 2^{<\omega}$ which is closed under initial segments. For any tree $T$, we let $[T]$ denote the set of all infinite paths through $T$. That is, $X \in[T]$ if and only if $\langle X(0), X(1), \ldots, X(n)\rangle \in T$ for all $n$. A $\Pi_{1}^{0}$ class is a set $P \subseteq 2^{\omega}$ for which there is a computable tree $T$ such that $P=[T]$. (Such classes are sometimes called computably bounded $\Pi_{1}^{0}$ classes in the literature.) Equivalently, $P \subseteq 2^{\omega}$ is a $\Pi_{1}^{0}$ class if and only if there is a primitive recursive tree $T$ such that $P=[T]$. The advantage of working with primitive recursive trees is that we can fix an effective enumeration of such trees and avoid any issues of partialness. Another useful equivalent definition for a $\Pi_{1}^{0}$ class $P$ is that $P=[V]$ for a $\Pi_{1}^{0}$ tree $V$. Such a tree is usually constructed as the intersection $V=\bigcap_{s} V_{s}$ of a nested uniform sequence $V_{0} \supseteq V_{1} \supseteq \cdots$ of computable trees.

2000 Mathematics Subject Classification. Primary 03D25, 03D35.

Key words and phrases. Logic, computability theory.

The author was partially supported by NSF Grant DMS-0400754.

(c)0000 (copyright holder) 
The main examples of $\Pi_{1}^{0}$ classes we will work with are separating classes. Given disjoint c.e. sets $A$ and $B$, the $\Pi_{1}^{0}$ class $\operatorname{Sep}(A, B)=\{D \mid A \subseteq D \wedge D \cap B=\emptyset\}$ is called the separating class of $\mathbf{A}$ and $\mathbf{B}$. If $A \cup B$ is coinfinite, then $\operatorname{Sep}(A, B)$ is a perfect $\Pi_{1}^{0}$ class and hence is uncountable. One of the main themes of this article is to transfer results from perfect $\Pi_{1}^{0}$ classes to classes of separating sets.

We work with propositional theories in the language with propositional variables $p_{i}, i \in \omega$. Let $S$ be a consistent c.e. propositional theory. The collection of complete consistent extensions of $S$ can be represented by a $\Pi_{1}^{0}$ class in the sense that there is a $\Pi_{1}^{0}$ class $P_{S}$ and a Turing degree preserving bijection between $P_{S}$ and the set of complete consistent extensions of $S$. We view each $X \in P_{S}$ as representing the theory generated by $\left\{p_{i} \mid i \in X\right\}$ and $\left\{\neg p_{i} \mid i \notin X\right\}$. Conversely, if we start with a $\Pi_{1}^{0}$ class $P$, then there is a c.e. theory $S_{P}$ and a Turing degree preserving bijection between the complete consistent extensions of $S_{P}$ and $P$. Furthermore, these processes are inverses in the sense that passing from a $\Pi_{1}^{0}$ class to a c.e. theory and back to a $\Pi_{1}^{0}$ class returns us to the original class. Therefore, studying complete consistent extensions of c.e. theories is equivalent to studying $\Pi_{1}^{0}$ classes. (For details of this correspondence, the reader is referred to Cenzer $[\mathbf{C}]$ and Cenzer and Remmel $[\mathbf{C R}]$.)

To examine this connection in more depth, we need additional notation. For $\sigma, \tau \in 2^{<\omega}$, we write $\sigma \sqsubseteq \tau$ if $\sigma$ is an initial segment of $\tau$. Similarly, we write $\sigma \sqsubseteq X$ if $\sigma$ is an initial segment of the set $X$. If $P$ is a $\Pi_{1}^{0}$ class, then $P[\sigma]$ denotes the set of all $X \in P$ such that $\sigma \sqsubseteq X$. If $T$ is a tree, then $T[\sigma]$ is the set of all $\tau \in T$ such that $\sigma \sqsubseteq \tau$. We sometimes abuse notation and treat $T[\sigma]$ as a tree, even though it is missing the proper initial segments of $\sigma$.

Definition 1.1. A $\Pi_{1}^{0}$ class $P$ is thin if for every $\Pi_{1}^{0}$ subclass $Q \subseteq P$, there is a finite set of strings $\sigma_{1}, \ldots, \sigma_{k}$ such that $Q=P\left[\sigma_{1}\right] \cup \cdots \cup P\left[\sigma_{k}\right]$. In other words, $Q$ is the intersection of $P$ with a clopen set in $2^{\omega}$.

Definition 1.2. Let $S$ be a consistent c.e. theory in the propositional language with variables $p_{i}, i \in \omega$.

(1) $S$ has few c.e. extensions if every c.e. extension $C$ of $S$ is a principal extension. That is, $C$ is generated by $S$ together with a single propositional formula.

(2) $S$ is essentially undecidable if $S$ has no decidable complete consistent extensions.

(3) $S$ is well generated if there are sets $A$ and $B$ such that $S$ is generated by $\left\{p_{i} \mid i \in A\right\}$ and $\left\{\neg p_{i} \mid i \in B\right\}$. (In this case, $A$ and $B$ must be c.e. and disjoint.)

The following properties of the correspondence between $\Pi_{1}^{0}$ classes and complete consistent extensions of c.e. theories follow from the definitions. First, $S$ has few c.e. extensions if and only if the corresponding $\Pi_{1}^{0}$ class is thin. Second, $S$ is essentially undecidable if and only if the corresponding $\Pi_{1}^{0}$ class has no computable members. Third, $S$ is well generated if and only if the corresponding $\Pi_{1}^{0}$ class is a separating class.

There are several different definitions for a Martin-Pour-El theory in the literature, each of which corresponds to some combination of these properties. The varying definitions were chosen to fit particular circumstances. For example, Cenzer, Downey, Jockush and Shore [CDJS] define a Martin-Pour-El theory to be 
a consistent c.e. theory with few c.e. extensions. Under their definition, MartinPour-El theories correspond to thin $\Pi_{1}^{0}$ classes. They chose this definition to fit their focus on countable $\Pi_{1}^{0}$ classes, each of which must contain a computable element and cannot be a class of separating sets for which $A \cup B$ is coinfinite. We will not work with this particular definition of a Martin-Pour-El theory.

In the survey papers Cenzer $[\mathbf{C}]$ and Cenzer and Remmel $[\mathbf{C R}]$, the authors define a Martin-Pour-El theory to be a consistent c.e. theory that is essentially undecidable and has few c.e. extensions. We refer to such theories as weak MartinPour-El theories. These theories correspond to perfect thin $\Pi_{1}^{0}$ classes with no computable paths. (It is known that the requirement of having no computable path is redundant and we give a proof of this fact in Section 2.) The construction of such a class is a basic example of the finite extension technique in $\Pi_{1}^{0}$ classes. (See Theorem 5.3 in Cenzer $[\mathbf{C}]$ for this construction.) Such a construction can be combined with other finite extension methods to build a perfect thin $\Pi_{1}^{0}$ class $P$ with extra properties such as $X^{\prime} \equiv_{T} X \oplus 0^{\prime}$ for all $X \in P$ (see Theorem 5.4 in Cenzer $[\mathbf{C}]$ ) or $X$ and $Y$ are Turing incomparable for any $X \neq Y \in P$ (use the finite extension construction in Theorem 4.7 in Jockusch and Soare [JSS]). Under the correspondence with c.e. theories, one obtains as immediate corollaries that there are weak Martin-Pour-El theories $T_{1}$ and $T_{2}$ such that every complete consistent extension of $T_{1}$ is generalized low (that is, satisfies $X^{\prime} \equiv_{T} X \oplus 0^{\prime}$ ) and every pair of distinct complete consistent extensions of $T_{2}$ is Turing incomparable.

Finally, in Downey [D], Downey, Jockusch and Stob [DJS] and Cholak, Coles, Downey and Herrmann [CCDH], a Martin-Pour-El theory is defined to be a consistent c.e. theory that is essentially undecidable, has few c.e. extensions and is well generated. We refer to these theories as strong Martin-Pour-El theories. These theories correspond to thin classes of separating sets with no computable paths. (As above, the condition of having no computable paths is redundant as long as the thin class of separating sets is infinite.) Such theories were first shown to exist by Martin and Pour-El [MP], giving rise to their name. In $[\mathbf{D}]$ and $[\mathbf{D J S}]$, the authors explore the possible Turing degrees of such theories, culminating in the classification that these degrees are exactly the array nonrecursive degrees. In each of the articles $[\mathbf{M P}],[\mathbf{D}]$ and $[\mathbf{D J S}]$, the authors construct such theories directly and obtain the corresponding $\Pi_{1}^{0}$ class results as (often unstated) corollaries.

One of the goals of this paper is to explore the possible degrees of complete consistent extensions of strong Martin-Pour-El theories by constructing thin classes of separating sets rather than directly constructing the theories themselves. We borrow a technique from Jockusch and Soare [JS ] to show that the basic finite extension construction of a perfect thin $\Pi_{1}^{0}$ class (as described in Cenzer $[\mathbf{C}]$ ) can be modified to give a thin class of separating sets. Once we build a thin class of separating sets using this modified finite extension construction, it will be almost immediately apparent that the construction can be combined with other (similarly modified) finite extension arguments. There are many such arguments in the literature and we mention two examples in Section 2.

These examples combine well known finite extension methods with the construction of a thin class of separating sets to yield the strong Martin-Pour-El theory analogues of the weak Martin-Pour-El theories $T_{1}$ and $T_{2}$ mentioned above. Although the modifications of known techniques required to get these results are not difficult, they do not appear to follow from previously published constructions of 
perfect thin $\Pi_{1}^{0}$ classes. Also, because Martin and Pour-El $[\mathbf{M P}]$, Downey $[\mathbf{D}]$ and Downey, Jockusch and Stob [DJS] directly construct their strong Martin-Pour-El theories rather than building the corresponding thin class of separating sets, it is not as immediately apparent how to combine their constructions with other finite extension arguments to yield these new results concerning the degrees of complete consistent extensions of strong Martin-Pour-El theories.

In Section 3, we examine the connection between a strong Martin-Pour-El theory and the set of positive and negative literals that generate it. Let $T$ be a strong Martin-Pour-El theory. We call $A=\left\{i \mid p_{i} \in T\right\}$ the set of positive literals in $T$, we call $B=\left\{i \mid \neg p_{i} \in T\right\}$ the set of negative literals in $T$ and we call $A \cup B$ the set of literals in $T$. Because the sets $A$ and $B$ are disjoint c.e. sets, $A \cup B$ has the same degree as both $A \oplus B$ and the theory $T$. Much of the previous work on strong Martin-Pour-El theories has focused on the degree of $A \cup B$ rather than on the degrees of $A$ and $B$. In this section, we examine the connection between the degrees of $A$ and $B$ and the degree of $A \cup B$. In particular, we show that it is possible for $T$ to have degree $0^{\prime}$ while both $A$ and $B$ have low degree. We also show it is possible to partition the set of literals $A \cup B$ into positive and negative halves in ways which result in different degrees for the complete consistent extensions of the corresponding theories. Specifically, we construct a sequence of strong MartinPour-El theories $T_{n}, n \in \omega$, such that each $T_{n}$ has the same set of literals (but divided differently into positive and negative halves) and no complete consistent extension of $T_{n}$ can compute a complete consistent extension of $T_{m}$ for $m \neq n$.

In Section 4, we consider limitations on the Turing degrees of elements of thin $\Pi_{1}^{0}$ classes (regardless of whether the $\Pi_{1}^{0}$ classes are classes of separating sets or not). We begin with a result of Cenzer, Downey, Jockusch and Shore [CDJS] which states that if $P$ is a thin $\Pi_{1}^{0}$ class and $X \in P$, then $X^{\prime} \leq_{T} X \oplus 0^{\prime \prime}$. We use a similar proof to derive a different restriction of the degrees of elements of thin $\Pi_{1}^{0}$ classes in terms of dominating functions and prove that this new condition is strictly stronger.

We assume that the reader is familiar with finite injury constructions in computability theory. The computability theoretic terminology and notation is standard and follows Soare $[\mathbf{S}]$. In particular, $\varphi_{e}$ denotes the $e^{\text {th }}$ partial computable function. The terminology for $\Pi_{1}^{0}$ classes follows Cenzer $[\mathbf{C}]$ and Cenzer and Remmel $[\mathbf{C R}]$. A node $\sigma$ on a tree $T$ is extendible if there is an infinite path $X \in[T]$ such that $\sigma \sqsubseteq X$. An extendible node $\sigma$ is called a branching node if both $\sigma * 0$ and $\sigma * 1$ are extendible. If $[T]=\operatorname{Sep}(A, B)$, then for each $n \in \omega$, either every extendible node of length $n$ is branching or no extendible node of length $n$ is branching. If all such nodes are branching, then we say $n$ is a branching level of $T$. Notice that the branching levels correspond exactly to the elements of the complement of $A \cup B$. Finally, we fix a effective enumeration $T_{e}, e \in \omega$ of all the primitive recursive trees.

\section{Thin separating classes}

In this section, we show how to extend some well known results for weak Martin-Pour-El theories to strong Martin-Pour-El theories. We begin with the observation that weak Martin-Pour-El theories correspond to perfect thin $\Pi_{1}^{0}$ classes. (See $[\mathbf{C C D H}]$ for a statement of this lemma in a slightly different context.) 
Lemma 2.1. A thin $\Pi_{1}^{0}$ class has no computable member if and only if it is perfect.

Proof. Let $P$ be a thin $\Pi_{1}^{0}$ class. If $P$ is not perfect, then it has an isolated path. Since any isolated path in a $\Pi_{1}^{0}$ class is computable, $P$ has a computable member. On the other hand, if $P$ has a computable path $X$, then $\{X\}$ is a $\Pi_{1}^{0}$ subclass of $P$. Therefore, $X$ is the sole member of $P$ intersected with a clopen set, which means $X$ is isolated in $P$ and hence is not perfect.

As mentioned in the introduction, weak Martin-Pour-El theories correspond to thin $\Pi_{1}^{0}$ classes with no computable paths, and therefore they correspond to perfect thin $\Pi_{1}^{0}$ classes. In this section, we show that many finite extension constructions for perfect thin $\Pi_{1}^{0}$ classes can be modified to build thin classes of separating sets with similar properties. Thus, they give corresponding results concerning strong Martin-Pour-El theories. (Notice that $\operatorname{Sep}(A, B)$ is perfect as long as $A \cup B$ is coinfinite.)

We begin with a basic construction of a thin class of separating sets. This construction is similar to the proof of the existence of a perfect thin $\Pi_{1}^{0}$ class given in Theorem 5.3 of Cenzer [C], but it also uses a technique from Jockusch and Soare [JS] to turn the $\Pi_{1}^{0}$ class into a class of separating sets. Although this particular construction is new, the result is an immediate corollary of the work of Martin and Pour-El [MP].

Theorem 2.2 (Martin, Pour-El). There are disjoint c.e. sets $A$ and $B$ such that $A \cup B$ is coinfinite and $\operatorname{Sep}(A, B)$ is thin.

Proof. Because we will use the same notation in the other constructions, we present it carefully here. We construct a $\{0,1\}$-valued partial computable function $\psi$ in stages and set $A=\{n \mid \psi(n)=1\}$ and $B=\{n \mid \psi(n)=0\}$. Letting $\psi_{s}$ denote the finite portion of $\psi$ constructed at stage $s$, we will have $\psi_{s} \subseteq \psi_{s+1}$ and $\psi=\cup_{s} \psi_{s}$. Define $A_{s}=\left\{n \mid \psi_{s}(n)=1\right\}$ and $B_{s}=\left\{n \mid \psi_{s}(n)=0\right\}$ and let $V_{s}=\operatorname{Sep}\left(A_{s}, B_{s}\right)$ denote the set of all finite binary strings which are compatible with $\psi_{s}$. That is,

$$
V_{s}=\left\{\sigma \in 2^{<\omega}|\forall n<| \sigma \mid\left(\psi_{s}(n) \downarrow \rightarrow \psi_{s}(n)=\sigma(n)\right)\right\} .
$$

Every node on $V_{s}$ is extendible and these trees form a uniformly computable nested sequence $V_{0} \supseteq V_{1} \supseteq \cdots$ such that $V=\bigcap_{s} V_{s}$ is a $\Pi_{1}^{0}$ tree for which $[V]=\operatorname{Sep}(A, B)$. (Each node on $V$ is also extendible. However, if we write $[V]=[T]$ for a computable tree $T$, then it is not necessarily the case that the extendible nodes of $T$ are even computable. We will return to this point later.)

To make the domain of $\psi$ coinfinite, we use movable markers $\delta_{s}(i)$ to denote the $i^{\text {th }}$ element of the complement of $A_{s} \cup B_{s}$ at stage $s$. That is, $\delta_{s}(0)<\delta_{s}(1)<$ $\delta_{s}(2)<\cdots$ and $\overline{A_{s} \cup B_{s}}=\left\{\delta_{s}(i) \mid i \in \omega\right\}$. We guarantee that $\lim _{s} \delta_{s}(i)=\delta(i)$ exists for each $i$.

To make $\operatorname{Sep}(A, B)=[V]$ thin, we meet the requirements

$$
R_{e}:\left[T_{e}\right] \subseteq[V] \Rightarrow \exists U\left(U \text { is clopen } \wedge\left[T_{e}\right]=[V] \cap U\right) .
$$

(Recall that $T_{e}$ is the $e^{\text {th }}$ primitive recursive tree.) We break each requirement into subrequirements $R_{e}^{\sigma}$ for each $\sigma \in V$ such that $|\sigma|=\delta(e)$. (These strings $\sigma$ are the nodes on $V$ at the $e^{\text {th }}$ branching level. Therefore, $R_{e}$ will have $2^{e}$ many subrequirements.) At stage $s$, we approximate these subrequirements by working 
with $R_{e}^{\sigma}$ for each $\sigma \in V_{s}$ such that $|\sigma|=\delta_{s}(e)$. Once $\delta_{s}(e)$ reaches its limit, we arrive at the final list of subrequirements. The subrequirements are

$$
R_{e}^{\sigma}: \sigma \in T_{e} \Rightarrow \forall \tau \sqsupseteq \sigma\left(\tau \in V \rightarrow \tau \in T_{e}\right) .
$$

Why do the subrequirements make $[V]$ thin? Suppose $\left[T_{e}\right] \subseteq[V]$ and let $\sigma_{0}, \ldots, \sigma_{k}$ be the strings such that $\sigma_{i} \in V \cap T_{e}$ and $\left|\sigma_{i}\right|=\delta(e)$. Because of $R_{e}^{\sigma}$, $\left[V\left[\sigma_{i}\right]\right] \subseteq\left[T_{e}\right]$ for $0 \leq i \leq k$, and for all other $\mu \in V$ with $|\mu|=\delta(e), \mu \notin T_{e}$ (and hence $\left.\left[T_{e}[\mu]\right] \cap[V]=\emptyset\right)$. Therefore, $\left[T_{e}\right] \subseteq[V]$ implies

$$
\left[V\left[\sigma_{0}\right] \cup V\left[\sigma_{1}\right] \cup \cdots \cup V\left[\sigma_{k}\right]\right]=\left[T_{e}\right]
$$

as required.

We prioritize the subrequirements by letting $R_{e}^{\sigma}$ have higher priority than $R_{i}^{\tau}$ if $e<i$ or if $e=i$ and $\sigma$ is less than $\tau$ in the lexicographic order. The construction has only finite injury and subrequirements for the same $R_{e}$ requirement will not injure each other.

At stage 0 , we set $\psi_{0}$ to be undefined everywhere. Assume we are at stage $s+1$ of the construction. If $\sigma \in V_{s}$ with $|\sigma|=\delta_{s}(e)$, then we say $R_{e}^{\sigma}$ needs attention if $\sigma \in T_{e}$ and

$$
\exists \tau \sqsupseteq \sigma\left(\tau \in V_{s} \wedge \tau \notin T_{e}\right) .
$$

(To make this condition effective, we look only at $e \leq s$ and $\tau$ with $|\tau| \leq s$. Because $T_{e}$ is a primitive recursive tree, we do not need to place any stage approximations on $T_{e}$.) If no subrequirement needs attention, then let $\psi_{s+1}=\psi_{s}$. Otherwise, let $R_{e}^{\sigma}$ be the highest priority requirement that needs attention and fix the corresponding string $\tau$. Define $\psi_{s+1}$ as follows: for all $i$ such that $|\sigma| \leq i<|\tau|$, let $\psi_{s+1}(i)=\tau(i)$, and for all other $j \in \operatorname{dom}\left(\psi_{s}\right)$, let $\psi_{s+1}(j)=\psi_{s}(j)$. Proceed to the next stage. This completes the description of the construction.

Notice what the action of $R_{e}^{\sigma}$ accomplishes. It does not define $\psi_{s+1}$ on any number of the form $\delta_{s}(k)$ for $k<e$, so these markers retain their values at stage $s+1$. All numbers $i$ such that $|\sigma| \leq i<|\tau|$ are now in $\operatorname{dom}\left(\psi_{s+1}\right)$, so $\delta_{s+1}(e) \geq|\tau|$. Because all the other numbers in the domain of $\psi_{s}$ keep their values, there is a unique string $\sigma^{\prime} \sqsupseteq \tau$ such that $\sigma^{\prime} \in V_{s+1}$ with length $\delta_{s+1}(e)$. The action for $R_{e}^{\sigma}$ has caused $R_{e}^{\sigma}$ to become the subrequirement $R_{e}^{\sigma^{\prime}}$ and since $\sigma^{\prime} \in V_{s+1}$ and $\sigma^{\prime} \notin T_{e}$ (since $T_{e}$ is a tree, $\tau \notin T_{e}$ and $\tau \sqsubseteq \sigma^{\prime}$ ), $R_{e}^{\sigma^{\prime}}$ is satisfied.

Each of the other $R_{e}$ subrequirements has undergone a similar change. That is, let $\mu$ be any other string on $V_{s}$ with $|\mu|=|\sigma|$ and let $\nu \sqsupseteq \mu$ be the string such that for all $|\mu| \leq i<|\tau|, \nu(i)=\tau(i)$. There is a unique string $\mu^{\prime} \sqsupseteq \nu$ such that $\mu^{\prime} \in V_{s+1}$ with length $\delta_{s+1}(e)$. (In fact, $\left|\mu^{\prime}\right|=\left|\sigma^{\prime}\right|$ and for all $|\sigma| \leq i<\left|\sigma^{\prime}\right|, \sigma^{\prime}(i)=\mu^{\prime}(i)$.) The subrequirement $R_{e}^{\mu}$ has become $R_{e}^{\mu^{\prime}}$. If $R_{e}^{\mu}$ had previously been satisfied in the sense that $\mu \notin T_{e}$, then $R_{e}^{\mu^{\prime}}$ is also satisfied since $\mu \sqsubseteq \mu^{\prime}$ and hence $\mu^{\prime} \notin T_{e}$ (since $T_{e}$ is a tree). Therefore, the action of $R_{e}^{\sigma}$ does not injure any previously satisfied $R_{e}$ subrequirement. Furthermore, because each number $i$ added to the domain of $\psi_{s+1}$ by $R_{e}^{\sigma}$ satisfies $i \geq|\sigma|=\delta_{s}(e)$, we have that $\delta_{s+1}(k)=\delta_{s}(k)$ for all $k<e$. Therefore, the action of $R_{e}^{\sigma}$ does not injure any subrequirement for $R_{i}$ with $i<e$. (The action may injure subrequirements for $R_{i}$ if $i>e$, but this injury is finitary.)

Finally, notice that the new function $\psi_{s+1}$ is consistent with $\psi_{s}$ in the sense that $\psi_{s} \subseteq \psi_{s+1}$. This follows because our chosen $\tau$ satisfies $\tau \in V_{s}$ and hence is compatible with $\psi_{s}$. Therefore, if $|\sigma| \leq i<|\tau|$ and $\psi_{s}(i) \downarrow$, we have $\psi_{s}(i)=\tau(i)$, so we have not changed the value of $\psi_{s}(i)$ at stage $s+1$. 
Corollary 2.3 (Martin, Pour-El). There is a strong Martin-Pour-El theory.

Proof. By Theorem 2.2, there is a thin class of separating sets $\operatorname{Sep}(A, B)$ such that $A \cup B$ is coinfinite. $\operatorname{Sep}(A, B)$ is perfect, so by Lemma 2.1, it has no computable members. The corresponding theory is a strong Martin-Pour-El theory.

The key property of the construction in Theorem 2.2 is that a subrequirement $R_{e}^{\sigma}$ which needs attention at stage $s$ can be satisfied by adding a finite segment above $\sigma$ (and above $\mu$ for each $\mu \in V_{s}$ with $|\mu|=|\sigma|$ ) to $V_{s}$ to form $V_{s+1}$. Furthermore, once $R_{e}^{\sigma}$ is satisfied, it is not injured by any other action which causes $R_{e}^{\sigma}$ to become $R_{e}^{\sigma^{\prime}}$ as long as $\sigma \sqsubseteq \sigma^{\prime}$. In particular, it is not injured by the later action of any $R_{i}^{\mu}$ for $i \geq e$. Therefore, we can build thin separating classes with other properties as long as these properties can be split into subrequirements of the form $S_{e}^{\sigma}$ (for $e \in \omega$ and $\sigma$ of length $\delta(e))$ each of which can be satisfied by adding a finite segment above $\sigma$ in a similar way and each of which is not injured by further extensions (by requirements of the form $S_{i}^{\mu}$ or $R_{i}^{\mu}$ for $i \geq e$ ) once it is satisfied.

We present two simple examples of this type of construction below. In each case, we specify the subrequirements $S_{e}^{\sigma}$ which will guarantee the property in question. We indicate how they can be satisfied using finite extensions and check that they are not injured by further finite extensions. We leave the remaining formal details of the finite injury constructions to the reader.

For the first example, we force every separating set to be generalized low. (A set $X$ is called generalized low if $X^{\prime} \equiv_{T} X \oplus 0^{\prime}$.) It is possible for every member of a $\Pi_{1}^{0}$ class to be low (that is, satisfy $X^{\prime} \equiv_{T} 0^{\prime}$ ), but this property cannot happen in an uncountable class since there are only countably many low sets. Therefore, attaining a property like generalized lowness is the best one can hope for in the context of $\operatorname{Sep}(A, B)$ where $A \cup B$ is coinfinite. The following theorem generalizes Theorem 5.4 in Cenzer $[\mathbf{C}]$ from perfect thin $\Pi_{1}^{0}$ classes to thin classes of separating classes.

THEOREM 2.4. There is a thin class $\operatorname{Sep}(A, B)$ such that $A \cup B$ is coinfinite and every $X \in \operatorname{Sep}(A, B)$ satisfies $X^{\prime} \equiv_{T} X \oplus 0^{\prime}$.

Proof. For each $e$ and each $\sigma$ of length $\delta(e)$, we add the following subrequirement to the construction in Theorem 2.2.

$$
S_{e}^{\sigma}: \varphi_{e}^{\sigma}(e) \downarrow \vee \forall \tau \in V\left(\sigma \sqsubseteq \tau \rightarrow \varphi_{e}^{\tau}(e) \uparrow\right)
$$

At stage $s$, we say that $S_{e}^{\sigma}$ needs attention (for $\sigma \in V_{s}$ with $|\sigma|=\delta_{s}(e)$ ) if $\varphi_{e, s}^{\sigma}(e)$ does not converge and there is a $\tau \in V_{s}$ such that $\sigma \sqsubseteq \tau$ and $\varphi_{e, s}^{\tau}(e)$ does converge. (Whenever we deal with convergent computations using a finite oracle such as $\tau$, we assume that the computation does not query any elements of the oracle which are larger than the length of the finite oracle.) If $S_{e}^{\sigma}$ is the highest priority subrequirement needing attention, then we define $\psi_{s+1}$ by $\psi_{s+1}(i)=\tau(i)$ for all $|\sigma| \leq i<|\tau|$ and $\psi_{s+1}(i)=\psi_{s}(i)$ for all $i$ in the domain of $\psi_{s}$. (Since $\tau \in V_{s}$, $\tau$ is consistent with $\psi_{s}$ and hence $\psi_{s} \subseteq \psi_{s+1}$.)

As in the previous construction, when $S_{e}^{\sigma}$ acts, it becomes $S_{e}^{\sigma^{\prime}}$ for a unique string $\sigma^{\prime} \sqsupseteq \sigma$ of length $\delta_{s+1}(e)$. Furthermore, if a future extension causes $S_{e}^{\sigma^{\prime}}$ to become $S_{e}^{\sigma^{\prime \prime}}$ for some $\sigma^{\prime \prime} \sqsupseteq \sigma^{\prime}$, then $S_{e}^{\sigma^{\prime \prime}}$ will remain satisfied since $\varphi_{e}^{\sigma^{\prime \prime}}(e)$ also converges.

To see why satisfying the $S_{e}^{\sigma}$ requirements is enough to establish the theorem, notice that $0^{\prime}$ can determine the final list of subrequirements $S_{e}^{\sigma}$ for any $e$ (uniformly 
in $e$ ). Furthermore, for any of these final subrequirements $S_{e}^{\sigma}, 0^{\prime}$ can determine whether $\varphi_{s}^{\sigma}(e)$ converges or not. Therefore, to calculate $X^{\prime}(e)$ we use $0^{\prime}$ to determine the final subrequirements $S_{e}^{\sigma}$, we use $X$ to determine which $S_{e}^{\sigma}$ subrequirement satisfies $\sigma \sqsubseteq X$, and we use $0^{\prime}$ again to determine whether $\varphi_{e}^{\sigma}(e)$ converges. Because $\varphi^{X}(e)$ converges if and only if $\varphi_{e}^{\sigma}(e)$ converges, we have calculated $X^{\prime}(e)$. Therefore, $X^{\prime} \leq_{T} X \oplus 0^{\prime}$ and hence $X^{\prime} \equiv_{T} X \oplus 0^{\prime}$.

We immediately get the following corollary which strengthens the well known result that there is a weak Martin-Pour-El theory in which every complete consistent extension $C$ satisfies $C^{\prime} \equiv_{T} C \oplus 0^{\prime}$.

Corollary 2.5. There is a strong Martin-Pour-El theory $S$ such that each complete consistent extension $C$ of $S$ satisfies $C^{\prime} \equiv_{T} C \oplus 0^{\prime}$.

Theorem 2.4 has some connection to work done by Cenzer, Downey, Jockusch and Shore $[\mathbf{C D J S}]$. They proved that if $T$ is a thin $\Pi_{1}^{0}$ class and the set of extendible nodes of $T$ is computable, then $X^{\prime} \equiv_{T} X \oplus 0^{\prime}$ for all $X \in[T]$. In the context of countable thin $\Pi_{1}^{0}$ classes, they also showed that it is possible to have the set of extendible nodes be computable and to even make the Cantor-Bendixson rank an arbitrarily large computable ordinal. However, for a class of separating sets $\operatorname{Sep}(A, B)$, if the set of extendible nodes is computable, then $A$ and $B$ must also be computable. To see why, let $T$ be such that $[T]=\operatorname{Sep}(A, B)$ and the set of extendible nodes of $T$ is computable. A number $n$ is in the complement of $A \cup B$ if and only there is some node at level $n$ of $T$ which has two immediate successors which are both extendible. (This situation says that $n$ can either be added to or kept out of the separating set and this situation can only occur when $n$ is in the complement of $A \cup B$.) However, if $A$ and $B$ are disjoint c.e. sets and the complement of $A \cup B$ is computable, then $A$ and $B$ are computable. By Lemma 2.1, if $A \cup B$ is coinfinite and $\operatorname{Sep}(A, B)$ is thin, then $A$ and $B$ cannot be computable. Therefore, it is not possible to obtain Theorem 2.4 by using a trick such as making the set of extendible nodes computable. Furthermore, it shows that making the set of extendible nodes computable is not the only way to make all the elements of a thin class satisfy $X^{\prime} \equiv_{T} X \oplus 0^{\prime}$.

Our second example has a slightly different feature which is best brought out by considering how a more classical finite extension argument works and how it differs from the constructions in the theorems so far. The standard construction of a perfect thin $\Pi_{1}^{0}$ class $P$ (as given in Cenzer $[\mathbf{C}]$ ) proceeds by building a uniform sequence of computable functions $\alpha_{s}: 2^{<\omega} \rightarrow 2^{<\omega}$ such that

- for all $\sigma, \alpha_{s}(\sigma * 0)$ and $\alpha_{s}(\sigma * 1)$ are incompatible extensions of $\alpha_{s}(\sigma)$,

- range $\left(\alpha_{s+1}\right) \subseteq$ range $\left(\alpha_{s}\right)$, and

- for all $\sigma, \lim _{s} \alpha_{s}(\sigma)=\alpha(\sigma)$ exists.

Let $V_{s}$ be the computable tree formed by taking the downward closure of range $\left(\alpha_{s}\right)$ and let $V=\bigcap_{s} V_{s}$ be the limiting $\Pi_{1}^{0}$ tree. To make $P=[V]$ thin, we meet the following subrequirements $S_{e}^{\sigma}$ for each $|\sigma|=e$.

$$
S_{e}^{\sigma}: \alpha(\sigma) \in T_{e} \rightarrow \forall \tau \sqsupseteq \sigma\left(\alpha(\tau) \in T_{e}\right)
$$

$S_{e}^{\sigma}$ requires attention at stage $s$ if $\alpha_{s}(\sigma) \in T_{e}$ and there is a $\tau \sqsupseteq \sigma$ such that $\alpha_{s}(\tau) \notin$ $T_{e}$. To satisfy $S_{e}^{\sigma}$, we define $\alpha_{s+1}$ so that $\alpha_{s+1}(\sigma)=\alpha_{s}(\tau)$ and $\alpha_{s+1}(\mu)=\alpha_{s}(\tau * \nu)$ for $\mu \sqsupseteq \sigma$ such that $\sigma * \nu=\mu$. This action does not cause $\alpha_{s+1}(\mu)$ to change for any $\mu \neq \sigma$ such that $|\mu|=|\sigma|=e$. Therefore, the subrequirements $S_{e}^{\sigma}$ for the same 
$e$ act independently of each other. This independence makes the construction more flexible but it also means that it does not necessarily build a class of separating sets.

In the construction for Theorem 2.2, the subrequirements for $R_{e}$ do affect each other, but they do not injure each other. The property in Theorem 2.4 of making the elements generalized low is typically forced using a standard finite extension argument like the one for a perfect thin $\Pi_{1}^{0}$ class. However, it can be adapted to work with the modified type of finite extension argument required to build a class of separating sets as shown above.

Not all finite extension arguments can be modified in this manner. The standard example of this phenomenon is the construction of a perfect $\Pi_{1}^{0}$ class $P$ for which each distinct pair of elements $X \neq Y \in P$ is Turing incomparable. In Theorem 4.7 of Jockusch and Soare [JS], the authors construct such a $\Pi_{1}^{0}$ class using a finite extension argument which can be easily combined with the construction of a thin perfect $\Pi_{1}^{0}$ class to yield a perfect thin class in which each pair of distinct elements is Turing incomparable. Such a class corresponds to the existence of a weak Martin-Pour-El theory for which any two distinct complete consistent extensions are Turing incomparable.

It is not possible to have such a property for a class of separating sets $\operatorname{Sep}(A, B)$ in which $A \cup B$ is coinfinite. In such a class, there are always separating sets $X$ and $Y$ such that $X \neq Y$ but the symmetric difference $X \triangle Y$ is finite. Such $X$ and $Y$ are Turing equivalent. However, Jockusch and Soare $[\mathbf{J S}]$ showed that this problem of finite symmetric differences is the only restriction by constructing $\operatorname{Sep}(A, B)$ such that $A \cup B$ is coinfinite and any pair of separating sets either has finite symmetric difference or is Turing incomparable. Because they did not give the details of the construction of such a class (as its existence is a modification of earlier techniques in the paper), we give the explicit construction below to illustrate that it is also a modified finite extension construction of exactly the type that can be combined with the thinness construction for separating classes as in Theorem 2.2. This combination of techniques yields a new theorem on thin classes of separating sets as well as a new result on strong Martin-Pour-El theories.

THEOREM 2.6. There exists a thin class $\operatorname{Sep}(A, B)$ such that $A \cup B$ is coinfinite and for any two sets $C, D \in \operatorname{Sep}(A, B)$, either $|C \triangle D|<\omega$ or $C$ and $D$ are Turing incomparable.

Proof. We add the following requirements for $e \in \omega$ to the construction in Theorem 2.2.

$$
S_{e}: \forall X, Y \in[V]\left(|X \triangle Y|=\omega \rightarrow \varphi_{e}^{X} \neq Y\right) .
$$

At stage $s$, we break this requirement into subrequirements $S_{e}^{\sigma}$ for each $\sigma \in V_{s}$ of length $\delta_{s}(e)$. The subrequirement $S_{e}^{\sigma}$ works to satisfy $S_{e}$ in the case when $\sigma \sqsubseteq X$.

We say that $S_{e}^{\sigma}$ needs attention at stage $s+1$ if it is not currently satisfied (this term is defined below) and there is a string $\mu \in V_{s}$ extending $\sigma$ (with length $\leq s)$ and an $m$ with $e \leq m \leq s$ such that $\varphi_{e, s}^{\mu}\left(\delta_{s}(m)\right)$ converges but it is not the case that $\varphi_{e, s}^{\mu}\left(\delta_{s}(m)\right)=\mu\left(\delta_{s}(m)\right)$. There are three ways this could happen: $\delta_{s}(m)$ is not in the domain of $\mu, \varphi_{e}^{\mu}\left(\delta_{s}(m)\right)$ converges to a number other than 0 or 1 , or $\varphi_{e}^{\mu}\left(\delta_{s}(m)\right)=1-\mu\left(\delta_{s}(m)\right)$. Once we act for $S_{e}^{\sigma}$, it is declared satisfied and it only becomes unsatisfied if some requirement of the form $S_{i}^{\tau}$ or $R_{i}^{\tau}$ acts with $i<e$. (The $R_{i}^{\tau}$ requirements are the thinness requirements of Theorem 2.2.) 
The action of $S_{e}^{\sigma}$ depends on the three possibilities listed above. First, if $\delta_{s}(m) \geq|\mu|$, then extend $\psi_{s}$ to $\psi_{s+1}$ by setting $\psi_{s+1}(x)=\mu(x)$ for all $x$ such that $|\sigma| \leq x<|\mu|, \psi_{s+1}\left(\delta_{s}(m)\right)=1-\varphi_{e}^{\mu}\left(\delta_{s}(m)\right)$, and $\psi_{s+1}(x)=\psi_{s}(x)$ for all other $x \in \operatorname{dom}\left(\psi_{s}\right)$. Since $\mu$ is compatible with $\psi_{s}$, this definition gives $\psi_{s+1} \supseteq \psi_{s}$ and no marker of the form $\delta_{s}(i)$ for $i<e$ has been added to $\operatorname{dom}\left(\psi_{s+1}\right)$. Therefore, the higher priority markers retain their values at stage $s+1$ and this action does not injure any higher priority requirements.

In this case, if $X$ is any extension of $\sigma$ which is in $\left[T_{s+1}\right]$, then $X$ extends $\mu$. Furthermore, $\varphi_{e}^{X}\left(\delta_{s}(m)\right)=\varphi_{e}^{\mu}\left(\delta_{s}(m)\right)$. However, for any $Y$ in $\left[T_{s+1}\right]$,

$$
Y\left(\delta_{s}(m)\right)=\psi_{s+1}\left(\delta_{s}(m)\right)=1-\varphi_{e}^{\mu}\left(\delta_{s}(m)\right) \neq \varphi_{e}^{\mu}\left(\delta_{s}(m)\right)=\varphi_{e}^{X}\left(\delta_{s}(m)\right) .
$$

Therefore, $\varphi_{e}^{X} \neq Y$ and we have satisfied $S_{e}^{\sigma}$.

Second, if $\varphi_{e}^{\mu}\left(\delta_{s}(m)\right)=1-\mu\left(\delta_{s}(m)\right)$, then we extend $\psi_{s}$ to $\psi_{s+1}$ as follows. For all $x$ such that $|\sigma| \leq x<|\mu|$, set $\psi_{s+1}(x)=\mu(x)$, and for all other $x \in \operatorname{dom}\left(\psi_{s}\right)$, set $\psi_{s+1}(x)=\psi_{s}(x)$. In particular, since $\delta_{s}(m)$ is in the domain of $\mu$ but is not in the domain of $\psi_{s}$, we have defined $\psi_{s+1}\left(\delta_{s}(m)\right)=\mu\left(\delta_{s}(m)\right)$. As above, we have $\psi_{s} \subseteq \psi_{s+1}$ and we have not changed the values of any higher priority markers.

Consider any extension $X$ of $\sigma$ in $\left[T_{s+1}\right]$ and any other set $Y \in\left[T_{s+1}\right]$. Since $X$ is an extension of $\sigma$ on $T_{s+1}$, it must be an extension of $\mu$. Therefore,

$$
\varphi_{e}^{X}\left(\delta_{s}(m)\right)=1-\mu\left(\delta_{s}(m)\right)=1-\psi_{s+1}\left(\delta_{s}(m)\right) \neq \psi_{s+1}\left(\delta_{s}(m)\right)=Y\left(\delta_{s}(m)\right) .
$$

So, we have satisfied the subrequirement $S_{e}^{\sigma}$.

Third, if $\varphi_{e}^{\mu}\left(\delta_{s}(m)\right)$ does not have the value 0 or 1 , then we extend $\psi_{s}$ to $\psi_{s+1}$ as follows. For all $x$ such that $|\sigma| \leq x<|\mu|$, let $\psi_{s+1}(x)=\mu(x)$, and for all other $x \in \operatorname{dom}\left(\psi_{s}\right)$, let $\psi_{s+1}(x)=\psi_{s}(x)$.

Consider any $X \in\left[T_{s+1}\right]$ extending $\sigma$. As above, $X$ must extend $\mu$, and therefore, $\varphi_{e}^{X}\left(\delta_{s}(m)\right)$ converges to a value other than 0 or 1 . Since $\varphi_{e}^{X}$ does not compute a set, we have satisfied the subrequirement $S_{e}^{\sigma}$.

In each of these three cases, when $S_{e}^{\sigma}$ acts it increases the value of $\delta_{s}(e)$ and becomes $S_{e}^{\sigma^{\prime}}$ where $\sigma^{\prime}$ is the unique string of length $\delta_{s+1}(e)$ such that $\sigma^{\prime} \sqsupseteq \mu \sqsupseteq \sigma$. It is clear from the descriptions above that if some other $S_{e}^{\tau}$ or $R_{e}^{\tau}$ requirement causes $S_{e}^{\sigma^{\prime}}$ to become $S_{e}^{\sigma^{\prime \prime}}$ for $\sigma^{\prime \prime} \sqsupseteq \sigma^{\prime}$, then $S_{e}^{\sigma^{\prime \prime}}$ stays satisfied.

It remains to verify that the construction works. Let $X, Y \in \operatorname{Sep}(A, B)$ be sets such that $|X \triangle Y|=\omega$ and assume for a contradiction that $\varphi_{e}^{X}=Y$. Since the symmetric difference is infinite, for all $n$, there is an $m>n$ such that $X(\delta(m)) \neq$ $Y(\delta(m))$. Pick a stage $s$ and an $m>e$ such that $\delta_{s}(m)=\delta(m)$, the subrequirements $S_{e}^{\sigma}$ have reached their limits, no requirement of higher priority acts after $s$, and $X(\delta(m)) \neq Y(\delta(m))$. Fix the string $\sigma$ such that $\sigma \sqsubseteq X$ and $S_{e}^{\sigma}$ is one of the final $S_{e}$ subrequirements. Fix a stage $t>s$ such that there is an $n<t$ for which $\varphi_{e, t}^{X \uparrow n}(\delta(m))$ converges.

Consider the action of $S_{e}^{\sigma}$ as stage $t+1$. Because no requirement of higher priority acts, $S_{e}^{\sigma}$ is free to act if it wants to. Let $\mu=X\left\lceil n\right.$. At this stage, $S_{e}^{\sigma}$ sees the convergent computation $\varphi_{e}^{\mu}(\delta(m))$. The only thing that would prevent $S_{e}^{\sigma}$ from acting at this stage (and hence being satisfied forever since no higher priority requirement injures it after this stage) is if $\varphi_{e}^{\mu}(\sigma(m))=\mu(\delta(m))$. However, in this case,

$$
\varphi_{e}^{X}(\delta(m))=X(\delta(m)) \neq Y(\delta(m)),
$$

which is a contradiction to the assumption that $\varphi_{e}^{X}=Y$. 
Combining all of the constructions so far, we get the following corollary.

Corollary 2.7. There is a strong Martin-Pour-El theory $S$ such that every complete consistent extension $C$ of $S$ satisfies $C^{\prime} \equiv_{T} C \oplus 0^{\prime}$ and every pair of distinct complete consistent extensions either differ on only a finite number of propositional variables or are Turing incomparable.

We end this section by pointing out one significant difference between perfect thin classes and thin classes of separating sets. Let $\mathcal{L}\left(2^{\omega}\right)$ denote the collection of $\Pi_{1}^{0}$ classes under inclusion. Cholak, Coles, Downey and Herrmann [CCDH] proved that the collection of perfect thin classes is definable in this structure while the collection of classes of separating classes is not. Furthermore, they proved that any two perfect thin classes are automorphic. In particular, automorphisms of $\mathcal{L}\left(2^{\omega}\right)$ can take a thin classes of separating sets to a perfect thin class which is not a class of separating sets.

\section{Degrees of literals and degrees of theories}

Another aspect of strong Martin-Pour-El theories that can easily be brought out using $\Pi_{1}^{0}$ classes is the connection between the degree of the theory $T$ and the degrees of the sets $A$ and $B$ generating the corresponding class of separating sets. Recall that if $T$ is a strong Martin-Pour-El theory, then the complete consistent extensions of $T$ correspond to the elements of $\operatorname{Sep}(A, B)$ where $A=\left\{i \mid p_{i} \in T\right\}$ (the positive literals of $T$ ) and $B=\left\{i \mid \neg p_{i} \in T\right\}$ (the negative literals of $T$ ). We call $A \cup B$ the set of literals of $T$. Downey [D] observed that $T \equiv_{T} A \oplus B \equiv_{T}$ $A \cup B$ (because $A$ and $B$ are disjoint c.e. sets). In this section, we investigate the relationship between the degree of $T$ and the degrees of $A$ and $B$. These results are related to the work of Downey, Jockusch and Stob [DJS] and we discuss these connections at the end of the section.

Definition 3.1. A c.e. set $C$ is called effectively simple if it is coinfinite and there is a computable function $f$ such that for every $e$, if $W_{e} \subseteq \bar{C}$, then $\left|W_{e}\right| \leq f(e)$.

Effectively simple sets are obviously noncomputable, but Martin $[\mathbf{M}]$ proved that they all have degree $0^{\prime}$. In the standard direct construction of a strong MartinPour-El theory as given in Downey [D], the degree of $T$ is $0^{\prime}$ because $A \cup B$ is effectively simple. We can easily add requirements to our earlier constructions of thin separating sets to make $A \cup B$ effectively simple. (Of course, this result immediately follows from Downey's construction via the correspondence between strong Martin-Pour-El theories and thin classes of separating sets.)

TheOrem 3.2 (Downey). There is a thin class of separating sets $\operatorname{Sep}(A, B)$ such that $A \cup B$ is effectively simple.

Proof. The effectively simple requirements (working with the function $f(e)=$ $2 e)$ are

$$
S_{e}: W_{e} \subseteq \overline{A \cup B} \Rightarrow\left|W_{e}\right| \leq 2 e .
$$

We say that $S_{e}$ needs attention at stage $s+1$ if $W_{e, s} \subseteq \overline{A_{s} \cup B_{s}}$ and there is a $k \geq 2 e$ such that $\delta_{s}(k) \in W_{e, s}$. If $S_{e}$ is the highest priority requirement needing attention, then we let $\psi_{s+1}\left(\delta_{s}(k)\right)=1$ and let $\psi_{s+1}(x)=\psi_{s}(x)$ for all $x$ in the domain of $\psi_{s}$. (That is, we put $\delta_{s}(k)$ into $A$.) There are no subrequirements to worry about here and once we act for $S_{e}$, it is never injured by the action of other requirements 
(even higher priority ones). Because $S_{e}$ acts in a finite extension manner, it can be added to any of the previous constructions.

COROLlary 3.3. There is a strong Martin-Pour-El theory $T$ such that $T \equiv_{T} 0^{\prime}$ and the generating sets $A=\left\{i \mid p_{i} \in T\right\}$ and $B=\left\{i \mid \neg p_{i} \in T\right\}$ have low degree.

Proof. This result follows from combining the effectively simple requirements with the proof of Theorem 2.4 to obtain disjoint c.e. sets $A$ and $B$ such that $A \oplus$ $B \equiv_{T} 0^{\prime}$ (and hence $T \equiv_{T} 0^{\prime}$ ) and such that every element of $\operatorname{Sep}(A, B)$ has generalized low degree. Since $A, \bar{B} \in \operatorname{Sep}(A, B)$ and $A, \bar{B} \leq_{T} 0^{\prime}$, it follows that $A$ and $B$ have low degree.

COROllary 3.4. There is a strong Martin-Pour-El theory $T$ such that no complete consistent extension of $T$ can compute $T$.

Proof. By combining the effectively simple requirements above with the requirements from Theorems 2.4 and 2.6, we get a thin class $\operatorname{Sep}(A, B)$ such that $A \cup B \equiv_{T} 0^{\prime}$ (and so $T \equiv_{T} 0^{\prime}$ ), $A$ and $B$ are low, and for any two separating sets $C$ and $D$, either $C \equiv_{T} D$ or $C$ and $D$ are Turing incomparable. Since $A, B<_{T} 0^{\prime}$, no separating set can have degree $\geq 0^{\prime}$. Furthermore, because any complete consistent extension of $T$ has the same degree as a separating set, none of these extensions can compute $0^{\prime} \equiv_{T} T$.

Next, we turn to the question of how the degrees of the complete consistent extensions of two strong Martin-Pour-El theories with the same set of literals are related. That is, suppose we have thin class of separating sets $\operatorname{Sep}(A, B)$ and $\operatorname{Sep}(C, D)$ such that $A \cup B=C \cup D$. Can separating sets from one class always compute separating sets for the other class? The answer turns out to be no.

Theorem 3.5. There are disjoint pairs of c.e. sets $A, B$ and $C, D$ such that $A \cup B=C \cup D$ is coinfinite, $\operatorname{Sep}(A, B)$ and $\operatorname{Sep}(C, D)$ are thin and for all $X \in$ $\operatorname{Sep}(A, B), Y \in \operatorname{Sep}(C, D), X$ and $Y$ are Turing incomparable.

Proof. We build two partial computable function $\psi$ and $\alpha$ in stages and let $A=\{n \mid \psi(n)=1\}, B=\{n \mid \psi(n)=0\}, C=\{n \mid \alpha(n)=1\}$ and $D=\{n \mid \alpha(n)=$ $0\}$. To make $A \cup B=C \cup D$, we guarantee that at all stages $s$, the domain of $\psi_{s}$ is equal to the domain of $\alpha_{s}$. Therefore, we can use one set of markers $\delta_{s}(n)$ to denote both $\overline{A_{s} \cup B_{s}}$ and $\overline{C_{s} \cup D_{s}}$.

The thinness requirements for the separating sets are met as in Theorem 2.2, although we now have the meet these requirements for both pairs $A, B$ and $C, D$. Therefore, we have two sets of requirements $R_{e}^{A, B}$ and $R_{e}^{C, D}$ analogous to the $R_{e}$ requirements of Theorem 2.2. We meet these requirements using the same strategy as before, except that when we extend $\psi_{s}$ to meet $R_{e}^{A, B}$, we also add all numbers in $\operatorname{dom}\left(\psi_{s+1}\right)-\operatorname{dom}\left(\psi_{s}\right)$ to the domain of $\alpha_{s+1}$.

More specifically, at stage $s$ the requirement $R_{e}^{A, B}$ breaks into subrequirements $R_{e}^{A, B, \sigma}$ for each $\sigma \in \operatorname{Sep}\left(A_{s}, B_{s}\right)$ with $|\sigma|=\delta_{s}(e)$. $R_{e}^{C, D}$ breaks into similar subrequirements. Since the markers $\delta_{s}(e)$ enumerate $\overline{A_{s} \cup B_{s}}=\overline{C_{s} \cup D_{s}}$, the strings used in the subrequirements for $R_{e}^{A, B}$ and $R_{e}^{C, D}$ have the same length, even though they might not be identical as strings. (That is, a number $i \in A_{s}$ may have been put into $D_{s}$ rather than into $C_{s}$.) $R_{e}^{A, B, \sigma}$ needs attention if $\sigma \in T_{e}$ and

$$
\exists \tau \sqsupseteq \sigma\left(\tau \in \operatorname{Sep}\left(A_{s}, B_{s}\right) \wedge \tau \notin T_{e}\right)
$$


(and similarly for a subrequirement of the form $R_{e}^{C, D, \mu}$ ). If $R_{e}^{A, B, \sigma}$ is the highest priority subrequirement needing attention, then we fix such a $\tau$ and define $\psi_{s+1}(i)=$ $\tau(i)$ and $\alpha_{s+1}(i)=\tau(i)$ for all $|\sigma| \leq i<|\tau|$ and for all other $j \in \operatorname{dom}\left(\psi_{s}\right)=$ $\operatorname{dom}\left(\alpha_{s}\right)$, we let $\psi_{s+1}(j)=\psi_{s}(j)$ and $\alpha_{s+1}(j)=\alpha_{s}(j)$. This action maintains $\operatorname{dom}\left(\psi_{s+1}\right)=\operatorname{dom}\left(\alpha_{s+1}\right)$ and does not cause any injury to a $R_{e}^{C, D, \mu}\left(\right.$ or $\left.R_{e}^{A, B, \nu}\right)$ subrequirement if $R_{e}^{C, D, \mu}$ (or $R_{e}^{A, B, \nu}$ ) was already satisfied.

To insure that the separating sets in $\operatorname{Sep}(A, B)$ and $\operatorname{Sep}(C, D)$ are Turing incomparable, we meet the requirements

$$
\begin{gathered}
S_{e}: \forall X \in \operatorname{Sep}(A, B)\left(\varphi_{e}^{X} \notin \operatorname{Sep}(C, D)\right) \text { and } \\
Q_{e}: \forall Y \in \operatorname{Sep}(C, D)\left(\varphi_{e}^{Y} \notin \operatorname{Sep}(A, B)\right) .
\end{gathered}
$$

These requirements break into subrequirements of the following form for each $\sigma \in$ $\operatorname{Sep}\left(A_{s}, B_{s}\right)$ and $\mu \in \operatorname{Sep}\left(C_{s}, D_{s}\right)$ with length $|\sigma|=|\mu|=\delta_{s}(e)$

$$
\begin{gathered}
S_{e}^{\sigma}: \forall X \in \operatorname{Sep}(A, B)\left(\sigma \sqsubseteq X \rightarrow \varphi_{e}^{X} \notin \operatorname{Sep}(C, D)\right) \text { and } \\
Q_{e}^{\mu}: \forall Y \in \operatorname{Sep}(C, D)\left(\mu \sqsubseteq Y \rightarrow \varphi_{e}^{Y} \notin \operatorname{Sep}(A, B)\right) .
\end{gathered}
$$

At stage $s+1$, we say that $S_{e}^{\sigma}$ needs attention if it is not already satisfied and

$$
\exists \tau \sqsupseteq \sigma\left[\tau \in \operatorname{Sep}\left(A_{s}, B_{s}\right) \wedge \varphi_{e}^{\tau}\left(\delta_{s}(e)\right) \downarrow\right]
$$

(and similarly for $Q_{e}^{\mu}$ ). If $S_{e}^{\sigma}$ is the highest priority requirement needing attention, then we fix such a $\tau$. Define $\psi_{s+1}(i)=\tau(i)$ for all $|\sigma| \leq i<|\tau|$ and $\psi_{s+1}(j)=\psi_{s}(j)$ for all other $j \in \operatorname{dom}\left(\psi_{s}\right)$. (If $\delta_{s}(e) \geq|\tau|$, also define $\psi_{s+1}\left(\delta_{s}(e)\right)=1$ so that $\delta_{s}(e)$ is in the domain of $\psi_{s+1}$.) Define $\alpha_{s+1}\left(\delta_{s}(e)\right)=1-\varphi_{e}^{\tau}\left(\delta_{s}(e)\right), \alpha_{s+1}(i)=\tau(i)$ for all $|\sigma| \leq i<|\tau|$ with $i \neq \delta_{s}(e)$ and $\alpha_{s+1}(j)=\alpha_{s}(j)$ for all other $j \in \operatorname{dom}\left(\alpha_{s}\right)$. We have again maintained that $\operatorname{dom}\left(\psi_{s+1}\right)=\operatorname{dom}\left(\alpha_{s+1}\right)$.

Consider any $X \in \operatorname{Sep}\left(A_{s+1}, B_{s+1}\right)$ such that $\sigma \sqsubseteq X$. As in the previous constructions, there is a unique $\sigma^{\prime} \in V_{s+1}$ such that $\sigma \sqsubseteq \tau \sqsubseteq \sigma^{\prime}$ and $\left|\sigma^{\prime}\right|=\delta_{s+1}(e)$. Since $\sigma \sqsubseteq X$, we have $\tau \sqsubseteq \sigma^{\prime} \sqsubseteq X$ and hence $\varphi_{e}^{X}\left(\delta_{s}(e)\right)=\varphi_{e}^{\tau}\left(\delta_{s}(e)\right)$. If $Y \in$ $\operatorname{Sep}\left(C_{s+1}, D_{s+1}\right)$, then

$$
Y\left(\delta_{s}(e)\right)=\alpha_{s+1}\left(\delta_{s}(e)\right)=1-\varphi_{e}^{\tau}\left(\delta_{s}(e)\right) \neq \varphi_{e}^{\tau}\left(\delta_{s}(e)\right)=\varphi_{e}^{X}\left(\delta_{s}(e)\right) .
$$

Therefore, we have successfully diagonalized to win $S_{e}^{\sigma}$. Future extensions by other $S_{e}, Q_{e}, R_{e}^{A, B}$ or $R_{e}^{C, D}$ subrequirements will not injure this action. (The actions taken for $Q_{e}^{\mu}$ are analogous.)

Given the previous constructions, it should be clear that these actions are successful, so we leave the remaining details to the reader.

Although we presented Theorem 3.5 in terms of two separating sets, a simple modification of the proof allows us to construct a countable sequence of theories with similar properties. (Of course, we could also make all separating sets have generalized low degree and all sets from the same $\operatorname{Sep}\left(A_{n}, B_{n}\right)$ be either Turing equivalent or Turing incomparable.)

THEOREM 3.6. There are disjoint pairs of c.e. sets $A_{n}, B_{n}$ for $n \in \omega$ such that for all $n \neq m, A_{n} \cup B_{n}=A_{m} \cup B_{m}, A_{n} \cup B_{n}$ is coinfinite, $\operatorname{Sep}\left(A_{n}, B_{n}\right)$ is thin and for all $X \in \operatorname{Sep}\left(A_{n}, B_{n}\right)$ and all $Y \in \operatorname{Sep}\left(A_{m}, B_{m}\right), X$ and $Y$ are Turing incomparable.

COROLlary 3.7. There is a sequence of strong Martin-Pour-El theories $T_{n}$ for $n \in \omega$ such that the set of literals in $T_{n}$ and $T_{m}$ are the same for any $n, m$ (and 
hence $\left.T_{n} \equiv_{T} T_{m}\right)$ and such that any complete consistent extension of $T_{n}$ cannot compute any complete consistent extension of $T_{m}$ for $m \neq n$.

Finally, we discuss two connections between these results and the work of Downey, Jockusch and Stob [DJS]. First, in [DJS], the authors classify the c.e. degrees of strong Martin-Pour-El theories as exactly the array nonrecursive degrees. However, Corollary 3.7 provides evidence that the degree of a Martin-Pour-El theory has little to do with the degrees of its complete consistent extensions. Therefore, while quite a bit is known about the possible degrees of strong Martin-Pour-El theories, this information does not tell us everything we might like to know about the possible collections of degrees of complete consistent extensions that can occur for these theories.

Second, in [DJS], the authors prove that a c.e. degree $\mathbf{d}$ is the degree of a strong Martin-Pour-El theory if and only if there are c.e. sets $A, B \leq_{T} \mathbf{d}$ such that $A \cup B$ is coinfinite and no set of degree $0^{\prime}$ separates $A$ and $B$. The example in Corollary 3.4 shows that in at least some examples, we can make the sets $A$ and $B$ be the sets of positive and negative literals for the theory $T$. Downey, Jockusch and Stob also proved that a c.e. degree $\mathbf{d}$ is the degree of a strong Martin-Pour-El theory if and only if there exist disjoint pairs of c.e. sets $A_{0}, B_{0} \leq_{T} \mathbf{d}$ and $A_{1}, B_{1} \leq_{T} \mathbf{d}$ such that the elements of $\operatorname{Sep}\left(A_{0}, B_{0}\right)$ are Turing incomparable with the elements of $\operatorname{Sep}\left(A_{1}, B_{1}\right)$. Corollary 3.7 shows that at least in some examples, we can make $A_{0} \cup B_{0}=A_{1} \cup B_{1}$ and make these sets be the generating sets of literals for strong Martin-Pour-El theories of the same degree.

\section{Limitations on thin classes}

The examples given so far illustrate that constructions of $\Pi_{1}^{0}$ classes which use the finite extension method can frequently be modified to yield similar (if not identical) results in the more restricted context of thin classes of separating sets. These examples might give the impression that there is little difference between the degrees of members of $\Pi_{1}^{0}$ classes and the degrees of members of thin classes (regardless of whether the thin class is a class of separating sets). However, there are important differences and we point out two such differences in this section. The first difference comes from Cenzer, Downey, Jockusch and Shore [CDJS].

Theorem 4.1 (Cenzer, Downey, Jockusch and Shore). If $X$ is an element of a thin $\Pi_{1}^{0}$ class, then $X^{\prime} \leq_{T} X \oplus 0^{\prime \prime}$.

It follows from Theorem 4.1 that there is no set $X \geq_{T} 0^{\prime \prime}$ such that $X$ is an element of a thin $\Pi_{1}^{0}$ class. Certainly, no such restriction applies in general $\Pi_{1}^{0}$ classes. Before giving the second condition, we restate the property that $X^{\prime} \leq_{T}$ $X \oplus 0^{\prime \prime}$ in terms of dominating functions. If $p$ is any partial function on $\omega$, we say that a total function $f$ strongly dominates $p$ if there is an $n$ for which $p(x) \leq f(x)$ for all $x \geq n$ such that $p(x)$ is defined.

Lemma 4.2. For any set $X$, the following are equivalent.

(1) $X^{\prime} \leq_{T} X \oplus 0^{\prime \prime}$.

(2) $\exists f \leq_{T} X \oplus 0^{\prime \prime} \forall e\left(f\right.$ strongly dominates $\left.\varphi_{e}^{X}\right)$.

(3) $\forall e \exists f \leq_{T} X \oplus 0^{\prime \prime}\left(f\right.$ strongly dominates $\left.\varphi_{e}^{X}\right)$.

Proof. To see that (1) implies (2), let $g \leq_{T} X^{\prime}$ be such that $g(e, n)=\varphi_{e}^{X}(n)$ if $\varphi_{e}^{X}(n)$ converges and $g(e, n)=0$ otherwise. Let $f \leq_{T} X^{\prime}$ be such that $f(n)=$ 
$\max \{g(e, n) \mid e \leq n\}+1$. By its definition, $f$ strongly dominates all $\varphi_{e}^{X}$, and by (1), $f \leq_{T} X \oplus 0^{\prime \prime}$.

(2) clearly implies (3), so we prove that (3) implies (1). Fix an index $e$ such that $X^{\prime}=W_{e}^{X}$ and let $g(m)$ be the least $s$ such that $m \in W_{e, s}^{X}$ if such an $s$ exists, and $g(m)$ is undefined otherwise. The partial function $g$ is computable from $X$, so fix a total function $f \leq_{T} X \oplus 0^{\prime \prime}$ which strongly dominates $g$. By altering a finite initial segment of $f$ (and hence not changing the degree of $f$ ), we can assume that $f(n) \geq g(n)$ for all $n$ on which $g(n)$ is defined. Let $h \leq_{T} X \oplus 0^{\prime \prime}$ be defined by $h(m)=\sum_{n<m} f(n)$. It follows that $W_{e, h(m)}^{X} \uparrow m=W_{e}^{X} \uparrow m$ for all $m$ and hence that

$$
X^{\prime}=W_{e}^{X} \leq_{T} X \oplus h \leq_{T} X \oplus 0^{\prime \prime}
$$

As an immediate corollary of Theorem 4.1 and Lemma 4.2 , we obtain the following.

Corollary 4.3. If $X$ is an element of a thin $\Pi_{1}^{0}$ class, then

$$
\exists f \leq_{T} X \oplus 0^{\prime \prime} \forall e\left(f \text { strongly dominates } \varphi_{e}^{X}\right) .
$$

For the second limitation on the degree of a member of a thin $\Pi_{1}^{0}$ class, we simplify the oracle needed to compute the strongly dominating function in Corollary 4.3 from $X \oplus 0^{\prime \prime}$ to $0^{\prime \prime}$. The proof of Theorem 4.4 is very similar to the proof of Theorem 4.1 .

THEOREM 4.4. If $T \subseteq 2^{<\omega}$ is a computable tree such that $[T]$ is a thin $\Pi_{1}^{0}$ class, then

$$
\exists f \leq_{T} 0^{\prime \prime} \forall e \forall X \in[T]\left(\varphi_{e}^{X} \text { is strongly dominated by } f\right)
$$

PROOF. It is straightforward to construct (uniformly in $e$ and $i$ ) computable trees $Q_{e}^{i} \subseteq 2^{<\omega}$ such that $\left[Q_{e}^{i}\right]$ is the $\Pi_{1}^{0}$ class of all $X$ for which $\varphi_{e}^{X}(i)$ diverges. Because $[T]$ is thin, $\left[Q_{e}^{i}\right] \cap[T]$ is a clopen subset of $[T]$. Therefore, for each $e$ and $i$, there is a level $n$ such that for each extendible node $\sigma \in T$ with $|\sigma|=n$, either $\varphi_{e}^{\sigma}(i)$ converges or else $\varphi_{e}^{\tau}(i)$ diverges for every extendible $\tau \in T$ with $\sigma \sqsubseteq \tau$. Using $0^{\prime \prime}$ as an oracle, we can find such a level $n$ and define $g(e, i)$ to be the maximum value of $\varphi_{e}^{\sigma}(i)$ for the extendible nodes $\sigma$ such that $|\sigma|=n$ and $\varphi_{e}^{\sigma}(i)$ converges. The function $f(i)=\max \{g(e, i) \mid e \leq i\}+1$ is the desired dominating function.

We end this paper by comparing the conditions on $X$ given in Theorems 4.1 and 4.4:

(C1) $X^{\prime} \leq_{T} X \oplus 0^{\prime \prime}$

(C2) $\exists f \leq_{T} 0^{\prime \prime} \forall e\left(\varphi_{e}^{X}\right.$ is strongly dominated by $\left.f\right)$.

In Lemma 4.5, we show that the nonuniform version of Condition (C2)

$$
\forall e \exists f \leq_{T} 0^{\prime \prime}\left(\varphi_{e}^{X} \text { is strongly dominated by } f\right)
$$

is already strong enough to imply Condition (C1). In Theorem 4.6, we show that Condition ( $\mathrm{C} 1$ ) is not strong enough to imply even the nonuniform version of Condition (C2) restricted to total functions

$$
\forall e \exists f \leq_{T} 0^{\prime \prime}\left(\varphi_{e}^{X} \text { total } \rightarrow \varphi_{e}^{X} \text { is dominated by } f\right. \text { ). }
$$

Therefore, although the proofs of Theorems 4.1 and 4.4 are very similar, the result of Theorem 4.4 is strictly stronger. 
LEMma 4.5. Let $X$ be a set such that for all e, there is an $f \leq_{T} 0^{\prime \prime}$ such that $\varphi_{e}^{X}$ is strongly dominated by $f$. Then $X^{\prime} \leq_{T} X \oplus 0^{\prime \prime}$.

Proof. Because $0^{\prime \prime} \leq_{T} X \oplus 0^{\prime \prime}$, this lemma follows trivially from the implication that Condition (3) implies Condition (1) in Lemma 4.2.

Theorem 4.6. There is a set $X$ such that $X^{\prime} \leq_{T} X \oplus 0^{\prime}$ (and hence $X^{\prime} \leq_{T}$ $\left.X \oplus 0^{\prime \prime}\right)$ and such that there is an index e for which $\varphi_{e}^{X}$ is total but not dominated by any function $\leq_{T} 0^{\prime \prime} .{ }^{1}$

To prove Theorem 4.6, we build a set $X$ with the following three properties.

(P1) $X^{\prime} \leq_{T} X \oplus 0^{\prime}$

(P2) there is a set $Y \leq_{T} 0^{\prime \prime}$ such that $Y<_{T} X$ and $X$ is c.e. in $Y$, and

(P3) $X \not_{T} 0^{\prime \prime}$.

To see that these three properties suffice, notice that by Property (P2), we can fix an index $e$ such that $X=W_{e}^{Y}$. Let $g(m)$ be the least $s$ such that $X \uparrow m=W_{e, s}^{Y} \uparrow m$. Since $Y<_{T} X, g$ is a total function computable from $X$. Suppose there is an $f \leq_{T} 0^{\prime \prime}$ such that $f$ dominates $g$. Since $f$ and $Y$ are both $\leq_{T} 0^{\prime \prime}, 0^{\prime \prime}$ can compute $W_{e, f(m)}^{Y}\lceil m$ for each $m$. However, for sufficiently large $m$ (past the point at which $f$ starts to dominate $g$ ), we have $X \uparrow m=W_{e, f(m)}^{Y} \uparrow m$. Therefore, $X \leq_{T} 0^{\prime \prime}$, contradicting Property (P3).

To meet Property (P1), we make $X$ 1-generic. A set $X$ is 1-generic if for every $e$, there is a finite initial segment $\sigma \sqsubseteq X$ such that either $\varphi_{e}^{\sigma}(e)$ converges or for every $\tau \sqsupseteq \sigma, \varphi_{e}^{\tau}(e)$ diverges. In the first case, we say that $\sigma$ forces $e$ into $X^{\prime}$ and in the second case, we say that $\sigma$ forces $e$ out of $X^{\prime}$. Every 1-generic set $X$ satisfies $X^{\prime} \leq_{T} X \oplus 0^{\prime}$, so we get Property (P1) immediately from this condition. (See Lerman $[\mathbf{L}]$ for a proof of this fact.) We let $J_{e}$ denote the requirement that $e$ is either forced into or out of $X^{\prime}$.

To meet Property (P2), we define $Y=\left\{2^{i} \cdot 3^{j} \mid i \in X \wedge 2^{i} \cdot 3^{j} \notin X\right\}$. Jockusch $[\mathbf{J}]$ has shown that for any 1-generic set $X$, the corresponding set $Y$ satisfies $Y<_{T} X$ and $X$ is c.e. in $Y$. Thus, we get most of the requirements of Property (P2) immediately from this result. We use a dumping strategy during the construction when injury occurs to insure that $Y \leq_{T} 0^{\prime \prime}$.

To meet Property (P3), we use a diagonalization strategy. For each $e$ we have a witness $x_{e}$ which may change during the construction due to injury by higher priority requirements but will stabilize at a finite stage. Initially we keep the witness $x_{e}$ out of $X$ and we wait for $\varphi_{e}^{0^{\prime \prime}}\left(x_{e}\right)$ to converge to 0 . (The construction uses $0^{\prime \prime}$ as an oracle, so we can recognize this convergence when it occurs.) If we see this convergence, then we put $x_{e}$ into $X$ to satisfy Property (P3). We let $D_{e}$ denote the diagonalization requirement that $X \neq \varphi_{e}^{0^{\prime \prime}}$.

We work with oracle $0^{\prime \prime}$ and at stage $s$, we specify a finite binary string $X_{s}$ as our current guess at the initial segment of $X$. There will be finite injury, meaning that initial segments of $X$ may change finitely often before settling down to be correct. Each $J_{e}$ requirement has a finite binary string parameter $\alpha_{e, s}$ which it uses to force $e$ into or out of $X^{\prime}$. Each $D_{e}$ requirement has a witness $x_{e, s}$ which it uses to diagonalize and has a binary parameter $i_{e, s}$ which denotes to $x_{e, s}$ th bit of

\footnotetext{
${ }^{1}$ Bjorn Kjos-Hanssen has pointed out that any 3-generic $X$ satisfies the conditions of Theorem 4.6 since one can show that the principal function of $X$ is not dominated by any function $\leq_{T} 0^{\prime \prime}$.
} 
$X_{s}$. The finite string $X_{s}$ will have the following form

$$
X_{s}=\alpha_{0, s} * i_{0, s} * \alpha_{1, s} * i_{1, s} * \cdots * \alpha_{s, s} * i_{s, s}
$$

where $\alpha_{k, s}$ is the current finite binary string parameter used by $J_{k}$ and $i_{k, s}$ is the binary digit used by $D_{k}$. The parameter $x_{k, s}$ denotes the position of the bit $i_{k, s}$. (We use $\sigma * \tau$ to denote the concatenation of the strings $\sigma$ and $\tau$ and we use $\sigma * i$ for $i \in\{0,1\}$ to denote the concatenation of $\sigma$ and $\langle i\rangle$.)

The basic strategy for $J_{e}$ is as follows. At stage $e$, we ask the oracle if there is a string $\sigma$ such that $\varphi_{e}^{X_{e} * \sigma}(e)$ converges. If so, we pick such a string $\sigma$ and let $\alpha_{e, e}=\sigma$. If not, we let $\alpha_{e, e}$ be the empty string. In the first case, we have forced $e$ into $X^{\prime}$ and in the second case, no matter how $X_{s}$ is extended in the future, we know $e \notin X^{\prime}$. This action involves only a finite extension of $X_{s}$ so it does not interfere with the higher priority strategies.

The basic strategy for $D_{e}$ is as follows. After $J_{e}$ has defined $\alpha_{e, e}$ at stage $e$, we let $x_{e, e}$ denote the length of $X_{e} * \alpha_{e, e}$ and set $X_{e+1}=X_{e} * \alpha_{e, e} * 0$. The trailing 0 is the bit $i_{e, e}$ which is initially set to 0 . As the construction continues, $D_{e}$ waits for a stage $s>e$ such that $\varphi_{e, s}^{0^{\prime \prime}}\left(x_{e, s}\right)$ converges to 0 . (The $0^{\prime \prime}$ oracle can recognize when this convergence occurs.) If this computation never converges to 0 , we win $D_{e}$ without any action. If this computation does converge to 0 , then we change $i_{e, s}$ to 1 (that is, we put $x_{e, s}$ into $X$ ), winning $D_{e}$. However, changing $i_{e, s}$ may injure the action taken by requirements $J_{k}$ for $e<k$ since we have caused a change below the length of the oracle that $J_{k}$ used to force $k$ into or out of $X^{\prime}$. This conflict is easily resolved by initializing all $J_{k}$ for $k>e$ and letting them force the jump again using finite extensions of the current approximation to $X$. (To make the construction easier to write, we also initialize the $D_{k}$ strategies for $k>e$, although any such strategy which is already satisfied is not injured by this action.)

The final piece of the construction is to take the set $Y$ into account. Although $Y$ is not defined until the end of the construction, we need to use a dumping strategy to make sure it will be computable from $0^{\prime \prime}$. We adjust the length of the strings $\alpha_{e, s}$ used by $J_{e}$ to make each $x_{e, s}$ be a prime number greater than 3 . The important point is that $x_{e, s}$ is never divisible by 2 or 3 , and hence the positions of the $i_{e, s}$ bits are never of the form $2^{i} \cdot 3^{j}$. (Numbers of this form are exactly the ones which could show up in $Y$.) Furthermore, when an element $x_{e, s}$ enters $X$ late for the sake of $D_{e}$, we place all numbers of the form $2^{x_{e, s}} \cdot 3^{j}$ which are less than $\left|X_{s}\right|$ into $X$ as well. We repeat this process of adding numbers to $X_{s}$ until we have closed under the property that if $a$ has been added to $X_{s}$, then all numbers of the form $2^{a} \cdot 3^{j}$ which are $<\left|X_{s}\right|$ have been added to $X$ as well. Because the numbers which are added are greater than $x_{e, s}$, they do not cause any additional injury. Furthermore, this action keeps any of these numbers $<\left|X_{s}\right|$ from entering $Y$ late because $x_{e, s}$ (or some other newly added number) is now in $X$. This action guarantees that $Y \leq_{T} 0^{\prime \prime}$.

We can now present the formal construction. During the construction, any parameter which is not explicitly redefined retains its value at the next stage. At stage 0 , ask if there is a string $\sigma$ such that $\varphi_{0}^{\sigma}(0)$ converges. If so, then let $\alpha_{0,0}=\sigma$ for the first such $\sigma$ for which $|\sigma|$ is a prime $>3$. If not, then let $\alpha_{0,0}$ be any string of length 5. In either case, let $X_{0}=\alpha_{0,0} * 0$. Set $x_{0,0}=\left|\alpha_{0,0}\right|$ and $i_{0,0}=X_{0}\left(x_{0,0}\right)=0$. 
At stage $s+1$, assume that we have defined a finite string $X_{s}$ and that it has the form

$$
X_{s}=\alpha_{0, s} * i_{0, s} * \alpha_{1, s} * i_{1, s} * \cdots * \alpha_{s, s} * i_{s, s}
$$

with $x_{e, s}$ equal to the position of $i_{e, s}$ for $e \leq s$. We first determine whether any of the diagonalization strategies $D_{e}$ for $e \leq s$ wants to act. Check if there is a witness $x_{e, s}$ such that $\varphi_{e, s}^{0^{\prime \prime}}\left(x_{e, s}\right)$ converges to 0 and $i_{e, s}=0$. If not, then we go immediately to forcing the jump by asking if there is a string $\sigma$ such that $\varphi_{s+1}^{X_{s} * \sigma}(s+1)$ converges. If so, then let $\alpha_{s+1, s+1}$ be the first such $\sigma$ for which $X_{s} * \sigma$ has prime length. If not, then let $\alpha_{s+1, s+1}$ be any string such that $X_{s} * \alpha_{s+1, s+1}$ has prime length. Set $X_{s+1}=X_{s} * \alpha_{s+1, s+1} * 0, x_{s+1, s+1}=\left|X_{s} * \sigma_{s+1, s+1}\right|$ and $i_{s+1, s+1}=X_{s+1}\left(x_{s+1, s+1}\right)=0$. Proceed to the next stage.

If there is an $e \leq s$ such that $\varphi_{e, s}^{0^{\prime \prime}}\left(x_{e, s}\right)$ converges to 0 and $i_{e, s}=0$, then let $e$ be the least such value. Let $Z$ denote the finite string of length $X_{s}$ which results from putting $x_{e, s}$ into $X_{s}$ and then closing under the property that if $k \in Z, k \notin X_{s}$ and $2^{k} \cdot 3^{j}<|Z|$, then $2^{k} \cdot 3^{j} \in Z$. Notice that all the numbers added to $X_{s}$ to form $Z$ are $\geq x_{e, s}$ and so $X_{s} \uparrow x_{e, s}=Z \uparrow x_{e, s}$. Let the parameters $\alpha_{j, s+1}$ and $x_{j, s+1}$ for $j \leq e$ retain their values from stage $s$. Let the parameters $i_{j, s}$ for $j<e$ retain their values from stage $s$ and let the parameter $i_{e, s+1}=Z\left(x_{e, s+1}\right)=Z\left(x_{e, s}\right)=1$.

Because this action may injure the earlier work of $J_{k}$ for $e<k$, we proceed by induction on $k$ for $e<k \leq s+1$ to meet $J_{k}$. We begin by forcing the jump on $e+1$. Let $Z^{\prime}=X_{s} \uparrow x_{e, s}=Z \uparrow x_{e, s}$ and let $\alpha^{\prime}$ be such that $Z=Z^{\prime} * 1 * \alpha^{\prime}$. Ask if there is a string $\sigma$ such that $\varphi_{e+1}^{Z * \sigma}(e+1)$ converges. If so, let $\alpha_{e+1, s+1}=\alpha^{\prime} * \sigma$ for the first such $\sigma$ for which $|Z * \sigma|$ is prime. If not, let $\sigma$ be any string such that $Z * \sigma$ has prime length and let $\alpha_{e+1, s+1}=\alpha^{\prime} * \sigma$. In either case, $Z * \sigma=Z^{\prime} * 1 * \alpha_{e+1, s+1}$. Let $x_{e+1, s+1}=\left|Z^{\prime} * 1 * \alpha_{e+1, s+1}\right|$ and define $X_{s+1}^{e+1}=Z^{\prime} * 1 * \alpha_{e+1, s+1} * 0$. Set $i_{e+1, s+1}=X_{s+1}^{e+1}\left(x_{e+1, s+1}\right)=0$. The set $X_{s+1}^{e+1}$ has the form

$$
X_{s+1}^{e+1}=\alpha_{0, s+1} * i_{0, s+1} * \alpha_{1, s+1} * i_{1, s+1} * \cdots * \alpha_{e+1, s+1} * i_{e+1, s+1}
$$

and $x_{j, s+1}$ denotes the position of $i_{j, s+1}$ for all $j \leq e+1$.

To continue the induction, assume that $e+1<k \leq s+1$ and $X_{s+1}^{k-1}$ has already been defined with its associated parameters so that

$$
X_{s+1}^{k-1}=\alpha_{0, s+1} * i_{0, s+1} * \alpha_{1, s+1} * i_{1, s+1} * \cdots * \alpha_{k-1, s+1} * i_{k-1, s+1}
$$

with $x_{j, s+1}$ denoting the position of $i_{j, s+1}$ for $j \leq k-1$. Ask if there is a string $\sigma$ such that $\varphi_{k}^{X_{s+1}^{k-1} * \sigma}(k)$ converges. If so, let $\sigma$ be the first such string for which the length of $X_{s+1}^{k-1} * \sigma$ is prime. If not, let $\sigma$ be any string such that the length of $X_{s+1}^{k-1} * \sigma$ is prime. In either case, set $\alpha_{k, s+1}=\sigma, x_{k, s+1}=\left|X_{s+1}^{k} * \alpha_{k, s+1}\right|$, $X_{s+1}^{k}=X_{s+1}^{k-1} * \alpha_{k, s+1} * 0$ and $i_{k, s+1}=X_{s+1}^{k}\left(x_{k, s+1}\right)=0$. The set $X_{s+1}^{k}$ has the form

$$
X_{s+1}^{k}=\alpha_{0, s+1} * i_{0, s+1} * \alpha_{1, s+1} * i_{1, s+1} * \cdots * \alpha_{k, s+1} * i_{k, s+1}
$$

so the induction can continue. Once $X_{s+1}^{s+1}$ is defined, set $X_{s+1}=X_{s+1}^{s+1}$ and end the stage.

This completes the description of the formal construction. We now verify that the construction succeeds.

LEMma 4.7. For each $k$, the parameters $\alpha_{k, s}, x_{k, s}$ and $i_{k, s}$ reach limits $\alpha_{k}, x_{k}$ and $i_{k}$. Furthermore, each $D_{k}$ requirement acts only finitely often. 
Proof. The key observation for this lemma was pointed out in the proof: when a strategy $D_{e}$ acts, it only places numbers into $X$ which are $\geq x_{e, s}$. Therefore, once the parameters $\alpha_{k, s}$ and $x_{k, s}$ are defined, they change only when a $D_{e}$ strategy with $e<k$ acts.

We proceed by induction on $k$. Assume by induction that the parameters $\alpha_{e, s}$, $x_{e, s}$ and $i_{e, s}$ for $e<k$ have reached their limits by stage $s$ and the $D_{e}$ strategies for $e<k$ have finished acting by stage $s$. By the observation above, the parameters $\alpha_{k, s}$ and $x_{k, s}$ do not change after stage $s$ and hence have reached their limits. After stage $s$, the initial segment $\alpha_{0} * i_{0} * \alpha_{1} * i_{1} * \cdots * \alpha_{k-1} * i_{k-1} * \alpha_{k}$ of $X$ is fixed. Also parameter $x_{k}$ denoting the length of this initial segment is fixed. The parameter $i_{k, s}$ will only change if $D_{k}$ acts to diagonalize. If this happens at stage $t \geq s$, then $i_{k, t}$ changes from 0 to 1 . $D_{k}$ will never want to act again since for any $u>t$, $i_{k, u}=X_{u}\left(x_{k}\right)=X\left(x_{k}\right)=1$. Therefore, $D_{k}$ acts at most once after stage $s$ and $i_{k, s}$ changes values at most once after stage $s$.

LEMMA 4.8. Each of the $D_{k}$ requirements is met.

PROOF. Let $t$ be a stage such that $\alpha_{e, t}$ and $x_{e, t}$ have reached their limits for $e \leq k, i_{e, t}$ has reached its limit for $e<k$ and the strategies $D_{e}$ for $e<k$ do not act after stage $t$. The value of the parameter $i_{k, s}$ is set to 0 at the last stage $s \leq t$ at which $x_{k, s}$ is changed. It retains the value 0 unless $\varphi_{e}^{0^{\prime \prime}}\left(x_{k}\right)$ converges to 0 . Therefore, if this convergence never occurs, the requirement $D_{k}$ is won. If this convergence does occur, then $D_{k}$ will be the highest priority requirement wanting to diagonalize and hence will change $i_{k, s}$ permanently to 1 . Therefore, $D_{k}$ is also won in this case.

LEMMA 4.9. $X$ is 1-generic.

Proof. Fix $e \in \omega$ and let $s+1$ be the last stage at which $\alpha_{e, s+1}$ is redefined. When $\alpha_{e, s+1}$ is defined, the initial segment $\widehat{X}=\alpha_{0} * i_{0} * \alpha_{1} * i_{1} * \cdots * \alpha_{e-1} * i_{e-1}$ is fixed. ( $\widehat{X}$ is either $X_{s}$ or $X_{s+1}^{e-1}$ depending on which case of the construction we use to define $\alpha_{e, s+1}$.) We define $\alpha_{e, s+1}=\alpha_{e}$ by searching for a string $\sigma$ which makes $\varphi_{e}^{\widehat{X} * \sigma}(e)$ converge. If there is such a string, then there is a $\sigma$ for which $\widehat{X} * \sigma$ has prime length. We define $\alpha_{e}=\sigma$ and have a correct initial segment $\widehat{X} * \alpha_{e}$ of $X$ for which $\varphi^{\widehat{X} * \alpha_{e}}(e)$ converges. If there is no such $\sigma$, then $\widehat{X}$ is a correct initial segment of $X$ for which $\varphi_{e}^{\tau}(e)$ diverges for all $\tau \sqsupseteq \hat{X}$. Therefore, $X$ satisfies the conditions for being 1-generic.

We next verify that Properties (P1)-(P3) hold for $X$.

Lemma 4.10. Property (P1) holds for $X$.

Proof. Any 1-generic set $G$ satisfies $G^{\prime} \leq_{T} G \oplus 0^{\prime}$. Therefore, this lemma follows immediately from Lemma 4.9 .

Lemma 4.11. Property (P3) holds for $X$.

ProOF. This follows immediately from Lemma 4.8 .

Finally, it remains to verify Property (P2). Fix a recursive functional $\Phi$ such that for any set $A, \Phi(A)=\left\{2^{i} \cdot 3^{j} \mid i \in A \wedge 2^{i} \cdot 3^{j} \notin A\right\}$. Jockusch $[\mathbf{J}]$ proved that if $G$ is 1-generic, then $\Phi(G)<_{T} G$ and $G$ is c.e. in $\Phi(G)$. Therefore, if we let 
$Y=\Phi(X)=\left\{2^{i} \cdot 3^{j} \mid i \in X \wedge 2^{i} \cdot 3^{j} \notin X\right\}$, then $Y<_{T} X$ and $X$ is c.e. in $Y$. To finish the verification of Property (P2), it remains to show that $Y \leq_{T} 0^{\prime \prime}$.

Lemma 4.12. $Y \leq_{T} 0^{\prime \prime}$.

Proof. To check if $2^{i} \cdot 3^{j}$ is an element of $Y$, run the construction of $X$ using $0^{\prime \prime}$ until a stage $s$ such that $\left|X_{s}\right|>2^{i} \cdot 3^{j}$. The lemma follows from the claim that

$$
2^{i} \cdot 3^{j} \in Y \Leftrightarrow i \in X_{s} \wedge 2^{i} \cdot 3^{j} \notin X_{s}
$$

To prove this equivalence, notice that for any $s$, once $X_{s}$ is defined, no number $<\left|X_{s}\right|$ is ever removed from $X$. Furthermore, if $a<\left|X_{s}\right|$ is added to $X$ at a stage $t>s$, then every number of the form $2^{a} \cdot 3^{j}$ which is $<\left|X_{t}\right|$ is also added to $X$ at stage $t$. Conversely, if a number of the form $2^{a} \cdot 3^{j}$ which is $<\left|X_{t}\right|$ is added to $X$ after stage $t$, then it must have been added because $a$ was also added to $X$ at the same stage.

To prove the $(\Leftarrow)$ direction, assume that $2^{i} \cdot 3^{j}<\left|X_{s}\right|, i \in X_{s}$ and $2^{i} \cdot 3^{j} \notin X_{s}$. Because we never remove elements $<\left|X_{s}\right|$ from $X, i \in X$. Furthermore, we would only add $2^{i} \cdot 3^{j}$ to $X$ after stage $s$ if we added $i$ to $X$ after stage $s$. However, $i$ is already in $X_{s}$, so it cannot be added to $X$ again later. Therefore, $2^{i} \cdot 3^{j} \notin X$ and we have that $2^{i} \cdot 3^{j}$ is in $Y$.

To prove the $(\Rightarrow)$ direction, assume that $2^{i} \cdot 3^{j}<\left|X_{s}\right|$ and $2^{i} \cdot 3^{j} \in Y$. Then, $i \in X$ and $2^{i} \cdot 3^{j} \notin X$. First, we claim that $i \in X_{s}$. Suppose not. Then, $i$ is added to $X$ at a stage $t>s$ and hence $2^{i} \cdot 3^{j}$ is also added to $X$ since $2^{i} \cdot 3^{j}<\left|X_{s}\right|<\left|X_{t}\right|$. Because numbers are never removed from $X$ once they have been added, we would have $2^{i} \cdot 3^{j} \in X$, which contradicts the fact that $2^{i} \cdot 3^{j} \notin X$. Therefore, $i \in X_{s}$. Second, we claim that $2^{i} \cdot 3^{j} \notin X_{s}$. This claim follows immediately from the fact that elements are never removed from $X$ once they are added and the fact that $2^{i} \cdot 3^{j} \notin X$.

This completes the proof of Theorem 4.6.

\section{References}

[C] D. Cenzer, $\Pi_{1}^{0}$ classes in computability theory, Handbook of computability theory, E.R. Griffor, ed., Elsevier, Amsterdam, 1999, pp. 37-85.

[CDJS] D. Cenzer, R. Downey, C. Jockush \& R.A. Shore, Countable thin $\Pi_{1}^{0}$ classes, Annals of Pure and Applied Logic, 59 (1993), 79-139.

[CR] D. Cenzer \& J.B. Remmel, $\Pi_{1}^{0}$ classes in mathematics, Handbook of recursive mathematics, Y. Ershov, S. Goncharov, A. Nerode \& J. Remmel, eds., North-Holland, Amsterdam, 1999, pp. 623-821.

[CCDH] P. Cholak, R. Coles, R. Downey and E. Herrmann, Automorphisms of the lattice of $\Pi_{1}^{0}$ classes: perfect thin classes and anc degrees, Transactions of the American Mathematical Society 353 (2001), 4899-4924 (electronic).

[D] R.G. Downey, Maximal theories, Annals of Pure and Applied Logic 33 (1987), 245-282.

[DJS] R. Downey, C. Jockusch \& M. Stob, Array nonrecursive sets of multiple permitting arguments, Recursion theory week (Proceedings Oberwolfach 1989), K. Ambos-Spies, G. Muller \& G. Sacks, eds., Springer-Verlag, Heidelberg, 1990, pp. 141-173.

[J] C.G. Jockusch Jr., Degrees of generic sets, Recursion Theory: Generalizations and Applications, F.R. Drake \& S.S. Wainer, eds., Cambridge University Press, Cambridge, 1980, 110-139.

[JS] C.G. Jockusch Jr. \& R.I. Soare, $\Pi_{1}^{0}$ classes and degrees of theories, Transactions of the American Mathematical Society 173 (1972), 33-56.

[L] M. Lerman, Degrees of unsolvability, Springer-Verlag, Heidelberg, 1983.

[M] D.A. Martin, Completeness, the recursion theorem and effectively simple sets, Proceedings of the American Mathematical Society 17 (1966), 838-842. 
[MP] D.A. Martin \& M.B. Pour-El, Axiomatizable theories with few axiomatizable extensions, Journal of Symbolic Logic 35 (1970), 205-209.

[S] R.I. Soare, Recursively enumerable sets and degrees, Springer-Verlag, Heidelberg, 1987.

Department of Mathematics, University of Connecticut U-3009, 196 Auditorium RoAd, Storrs, CT 06269

E-mail address: solomon@math.uconn.edu 